\title{
Application of Latex in Teaching and Scientific Research for Chinese under International Environment
}

\author{
Linli Fan, Li Liu* \\ School of Information Science and Engineering, Dalian Polytechnic University, \\ Dalian, China \\ *Corresponding author e-mail: liu.li@dlpu.edu.cn
}

\begin{abstract}
LaTeX is an international typesetting system widely used in writing and editing documents of teaching and scientific research work. It was originally developed as an English oriented automatic system, but it is now possible to identify Chinese by developing designs. The main feature of LaTeX is the ability to quickly process complex mathematical formulas and tables with simple statements. Besides, LaTeX specifies the hierarchy of whole article, users can process each hierarchical structure at one time, and directly generate stable PDF documents. LaTeX, due to its stable and intelligent layout function, plays more and more important role in the exchange and cooperation of international teaching and research area.
\end{abstract}

KEYWORDS: LaTeX typesetting system, International exchange and cooperation, Teaching and research

\section{Introduction}

The LaTeX typesetting system was developed by American mathematicians and computer scientists in the early 1980s, basis on the Tex of Donald E. Knuth professor at Stanford University [1]. LaTeX uses Tex as formatting engine, which is more convenient than the Tex system. LaTeX makes it easier and simpler for users to use Tex packages, and it is suitable for multiple operating systems.

LaTeX system [2] is a professional typesetting tool in the fields of mathematics, physics, computer science and technology; other areas such as chemistry, biology, engineering, and linguistics also have a large number of users. In recent years, LaTeX has been widely used in academic journals, especially in the fields of mathematics and physics. LaTeX system has powerful logic structure and standard formula typesetting, and can realize formula automatic numbering.

This system was originally developed as an English-oriented automatic typesetting system, but it is now possible to identify Chinese by developing 
designs. LaTeX usually has two ways to deal with Chinese, one is to use the CCT system developed by the Institute of Computational Science and Scientific Engineering Computing of the Chinese Academy of Sciences, and the other is to use the CJK macro package [3]. When contributing to international journals, we can directly use the given LaTeX macro package to write articles. Using simple sentences to realize recognition and handover of LaTeX system in English and Chinese, and run steadily in different systems. Therefore, it is more conducive to strengthening international cooperation and exchanges in scientific research.

As a professional high quality typesetting system, LaTeX has obvious advantages in typesetting of science and technology periodicals. Compared with other typesetting software such as Word, WPS and Founder, LaTeX has better effect in character quality, scientific articles and mathematical formula typesetting. For example, in teaching and research, writing articles is a common problem, and not only to the content of the article, but also the overall layout of the article. If choose to write a paper with Word, one should pay attention to the whole structure of the article and adjust it at any time while perfecting the content of the article. But by using LaTeX, there is no need to consider the format. After compiling, LaTeX can be automatically typesetted at any time, allowing users to focus on the content of the document. LaTeX provides convenience for the work of university teachers and researchers, and enables better communication and cooperation with international research fields. The application of LaTeX should attract extensive attention.

\section{Comparison between LaTeX and Word}

Word text editing software is widely used because of its strong visibility. In most universities in China, the number of text editors using LaTeX automatic typesetting system is relatively small. Word is very convenient for easy-to-type articles, but it is inconvenient to write a complicated article, such as a graduation thesis or some complicated articles with formulas and charts.

LaTeX automatic typesetting system is better than Word typesetting, for example, Word is usually encounter a problem of inconsistent document format. Even if using the full-selection and unified formatting function, for special fonts or formats there is still a need for comparison and manual typesetting. However, LaTeX typesetting system can be written and hierarchically designed through templates, which has strong logic and does not require manual formatting. If need to enter a special character, the specified simple statement for arbitrary combination can be used, and after compiling texts will automatically displayed in the specified location of whole article.

Inserting an image into a word document, the size, shape and location of the picture need to be adjusted. If there are multiple pictures, the size and location of the images can hardly be consistent. However, LaTeX system can set the size and position of the pictures by simple code design, and all the pictures are in the same format and automatically numbered. There will be no inconsistency in the position 
and size of the picture in the article, and to cite the picture is very convenient and fast. Insert picture code design example is as follows:

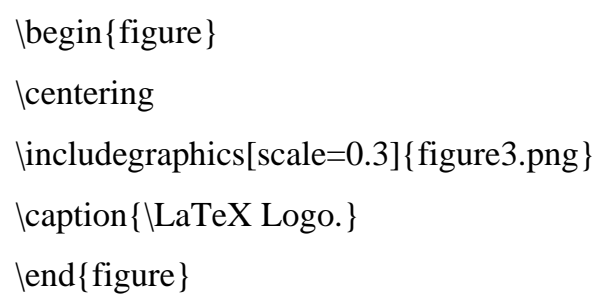

Insert a picture named figure3.png in the article, use centering to ensure that picture is centered, the size of the picture is limited by scale $=0.3$, caption is used to name picture. The effect of the picture in the article is shown in Figure 1.

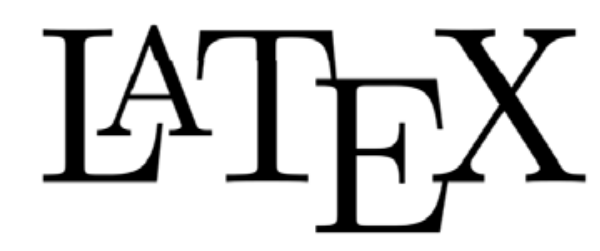

FIG. 1. LTEXLogo.

We can make figures bigger or smaller by scaling them. Figure1 has been scaled by $30 \%$.

Figure. 1 Insert pictures with LaTeX typesetting system

Every article has headlines and text at all levels, but the font format of headlines and text at all levels is not consistent in Word, so it has to format the headlines separately. In LaTeX, the title is written by the code section, just write the title to brackets, and if there are subheadings, add subsection after the title.

In generally, Word documents can generate directories automatically, but format needs to be adjusted. When the article title needs to be modified, the title name or page number in the catalog should be changed, and the catalog needs to be regenerated and formatted, in this way the workload of users is increased. However in LaTeX, the directory is updated automatically according to the content of the article, and the format is set by the template, so it doesn't need to be adjusted repeatedly. 


\section{Application of LaTeX in teaching and scientific research for Chinese}

LaTeX intelligent typesetting system can design templates, or directly use templates. So LaTeX has many uses, such as writing academic papers, writing test papers, applying in journals, making PPT, etc.

\subsection{Application of LaTeX system in academic papers}

Writing academic papers is an important part of students, and is a summary of knowledge. The typesetting of academic papers emphasizes the standardization of format, formulae, charts and references are difficult in academic papers [4]. For example, graduation thesis as the final document of graduation project is very complex, contains a large number of pictures and formulas, the format requirements are very strict. Some Chinese universities will make corresponding paper templates according to the characteristics of their own schools and national regulations. It is extremely convenient to write graduation thesis with $\mathrm{LaTeX}$, and students will not repeatedly check and modify the format. Using LaTeX, it can quickly implement complex formulas by typing simple code, greatly save the editing time of paper, meanwhile the standardization of paper format is strengthened.

\subsection{Application of LaTeX system in test paper editing}

The questions in the examination paper are complex, including choice questions, blank-filling questions, judgment questions, subjective questions (simple answer questions, discussion questions, calculation questions), etc. After compiling, we also need to give the corresponding reference answers. In the process of compiling, we should determine the actual solution space needed by the test questions according to the length of the answers. LaTeX can customize the typesetting environment according to given macro commands and actual requirements to achieve high quality typesetting results. Therefore, using the template designed by LaTeX to compile the test paper, only need to fill in the corresponding content according to the macro command to complete the test paper production. Using LaTeX to edit source code and generated test paper, it is easy to transmit and store, and are convenient for teachers to discuss papers and teaching contents.

\subsection{Application of LaTeX system in Journal submission}

LaTeX is an intelligent typesetting system for international submissions. The commonly used document classes include article, report, book, paper and thesis. The basic syntax is as follows:

Idocumentclass \{article\}

Idocumentclass \{report\} 
All kinds of periodicals have a fixed format typesetting requirements, some foreign periodicals will be directly given LaTeX typesetting format macro file. The font, font size, header size and chart formula of the article are all set by macro file, only need to be introduced directly. Modification does not need to consider formula and chart numbering. LaTeX can be automatically sorted and added. In addition, when LaTeX is written, it can use notations (\%) to add annotations to facilitate marking and modification. In particular, in the course of the exchange of articles, LaTeX source code can be used to communicate with researchers at home and abroad, greatly improving the efficiency of international cooperation.

\subsection{Transformation between LaTeX and Word}

In the process of using Word to write articles, one may encounter the problem of typesetting errors. If re-typesetting, it will take a lot of work. LaTeX typesetting system and word can be converted to each other [5]. Both typesetting systems have their advantages. LaTeX is preferred by scientists and technicians for typesetting high-quality scientific books or papers, especially those containing a large number of mathematical formulas. The combination of the two typesetting systems can reduce workload and improve efficiency.

\section{Conclusion}

In teaching and scientific research, it is usually necessary to write scientific documents to display and popularize academic achievements. A good article needs not only high-quality content but also standardized typesetting. Only a combination of content and layout can form a successful article. As a powerful typesetting system, LaTeX is widely used in teaching and scientific research for its independent content and format. LaTeX can be used in multilingual environments with the development of LaTeX in recent decades and with the development of LaTeX by researchers. LaTeX facilitates international cooperation and exchanges in teaching and research, and promotes academic cooperation for Chinese and abroad. At the same time, it helps to introduce advanced technology, promote cultural integration and broaden horizons.

\section{Acknowledgments}

Authors thank for the financial support by the educational reform project number 61001706.

\section{References}

[1] Sanjailal K P. LATEX: A high quality document preparation system for scientific and technical literature [J]. Cftri, 2012. 
Frontiers in Educational Research

ISSN 2522-6398 Vol. 1, Issue 1: 1-6, DOI: 10.25236/FER.030001

[2] Luo Z. Design and realization of degree thesis template based on LATEX system [J]. Journal of Shenyang Institute of Aeronautical Engineering, 2007.

[3] Jiang Z L. Compilation of Chinese TeX Text Files by Use of CJK Packages [J]. Acta Scientiarum Naturalium Universitatis Sunyatseni, 2004.

[4] Yu Y.L., Zhang L.L.. The LaTeX Software in Academic Typesetting [J]. Vaule Engineering, 2012.

[5] Stone W R. Converting Between Tex or LaTeX and Word [From the Screen of Stone] [J]. IEEE Antennas \& Propagation Magazine, 2017. 